\title{
Is the graviton a domain wall glueball?
}

\author{
Richard C. Brower ${ }^{a *}$ \\ a Physics Department \\ 590 Commonwealth Avenue \\ Boston University \\ Boston MA 02215
}

Strong coupling calculations for the glueball spectrum in an $A d S^{7}$ black hole are modified by introducing an UV cut-off at a Planck brane. A new normalizable state in the tensor spectrum is found which reproduces Einstein-Hilbert gravity up to exponentially small corrections due to off shell mixing with massive glueballs.

\section{INTRODUCTION}

Recent developments in string theory have changed our perspective on the ancient puzzle on how to construct the QCD string. The conventional wisdom had been that the fundamental superstring for gravity operates exclusively at or near the the Planck scale with the extra 6 dimension compactified in Planckian units. The standard model (and therefore QCD) would appear as simply a low energy effective field theory long after all stringy effects have disappeared. In this scenario the fact that confinement of chromodynamic flux and the large $\mathrm{N}$ expansion might also exhibit stringy features is essentially accidental, with no physical relation to the strings of quantum gravity.

With the discovery of Maldacena's string/gauge duality [1], we now have explicit examples of the equivalence between 10-d superstring and 4-d Yang-Mills theory. The key new ingredient is a strongly "warped" radial (or 5-th) dimension as illustrated in the weak/strong duality between strings in $A d S^{5} \times S^{5}$ and 4-d $\mathcal{N}=4$ super Yang Mills. The redshift in the warped axis interpolates between low energy (IR) and high energy (UV) physics. Extensions of the string/gauge duality have been suggested by Witten 2 and others for non-SUSY QCD like theories exhibiting discrete glueball spectra, con-

\footnotetext{
*This work was supported in part by the Department of Energy under Contracts No. DE-FG02-91ER40676
}

finement, etc. In addition Randall and Sundram have shown how brane world gravity maybe realized in AdS space with the Planck/TeV hierarchy separated exponentially as function of the proper distance in the warped axis.

Here I review our recent calculations of the glueball spectrum at strong coupling [3] and its extension to include brane world gravity 4 . The goal is to develop a toy model which is illustrative of this new approach to the QCD string.

\section{GLUEBALLS IN ADS BLACK HOLE}

The $Q C D_{4}$ model proposed by Witten [2] modifies the 11-d $A d S^{7} \times S^{4}$ metric solution to Mtheory or its low energy limit 11-d supergravity,

$$
\begin{aligned}
S & =\frac{1}{2 \kappa_{11}} \int d^{11} x \sqrt{-g_{11}}\left(R_{11}-\left|F_{4}\right|^{2}\right) \\
& -\frac{1}{12 \kappa_{11}} \int A_{3} \wedge F_{4} \wedge F_{4}+\text { fermions }
\end{aligned}
$$

by compactified both the 11-th axis reducing it to IIA string theory and a second "thermal" (or $\tau$ ) axis with anti-periodic Fermionic boundary conditions to break the conformal and super symmetries. The resultant background metric is an $A d S^{7} \times S^{4}$ black hole,

$d s^{2}=\frac{1}{r^{2}-r^{1-d}} d r^{2}+\left(r^{2}-r^{1-d}\right) d \tau^{2}+r^{2} \eta_{\mu \nu} d x^{\mu} d x^{\nu}$

The minimum value $r>r_{\min }=1$ is a co-ordinate singularity at the black hole horizon. 

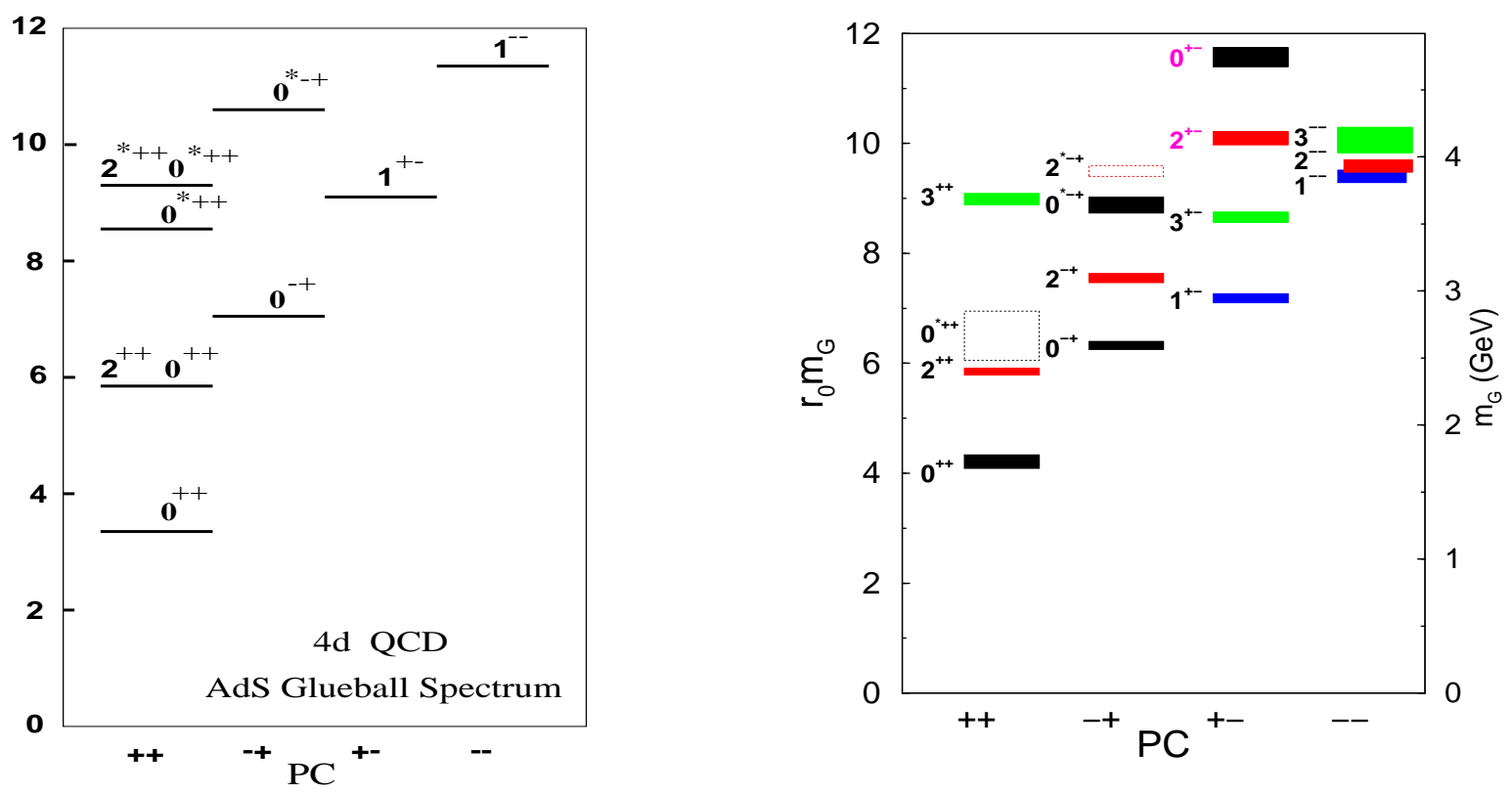

Figure 1. Comparison of AdS glueball spectrum for $Q C D_{4}$ in strong coupling (left) and the Morningstar $\&$ Peardon lattice spectrum (right). The scales are adjusted to fit $2^{++}$with $1 / r_{0}=410 \mathrm{Mev}$.

In the strong coupling 'tHooft limit $\left(g^{2} N \rightarrow\right.$ $\infty)$, the inverse QCD string tension vanishes like $\alpha^{\prime} \sim\left(R_{a d s} / r_{\text {min }}\right)^{3} \alpha_{\text {string }}^{\prime} \sim 1 /\left(g^{2} N \Lambda^{2}\right)$, where $\Lambda$ sets the scale of the glueball spectrum. Hence the string collapses to a point so the glueball spectrum can be found as the normal modes in the supergravity fields $G_{M N}, A_{M N L}$ (see Table below).

\begin{tabular}{|c|c|c|c|}
\hline$G_{\mu \nu}$ & $G_{\mu, 11}$ & $G_{11,11}$ & $m_{0}$ \\
\hline$G_{i j}$ & $C_{i}$ & $\phi$ & \\
$2^{++}$ & $1_{(-)}^{++}$ & $0^{++}$ & 4.7007 \\
\hline$G_{i \tau}$ & $C_{\tau}$ & & \\
$1_{(-)}^{-+}$ & $0^{-+}$ & & 5.6555 \\
\hline$G_{\tau \tau}$ & & & \\
$0^{++}$ & & & 2.7034 \\
\hline \hline$A_{\mu \nu, 11}$ & $A_{\mu \nu \rho}$ & & $m_{0}$ (Eq.) \\
\hline$B_{i j}$ & $C_{123}$ & & 7.3059 \\
$1^{+-}$ & $0_{(-)}^{+-}$ & & \\
\hline$B_{i \tau}$ & $C_{i j \tau}$ & & 9.1129 \\
$1_{(-)}^{--}$ & $1^{--}$ & & \\
\hline
\end{tabular}

The entire strong coupling glueball spectrum that lies in the superselection sector of $Q C D_{4}$, has been calculated [3] and its comparison with the numerical values determined in lattice QCD (Fig. 1) reveals surprisingly good agreement in view of the extreme approximation of the strong coupling limit.

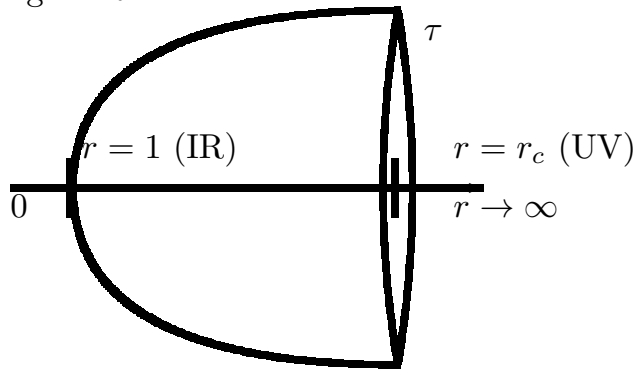

All the lowest $J^{P C}$ states are present, requiring contributions from all the bosonic fields in IIA supergravity. Even the radial excitations (illustrated by $0^{*-+}$ in Fig. 1) are the right order of magnitude. The absence of higher spins, such as $3^{+ \pm}$, is a direct consequence of the strong coupling (or infinite tension) limit whereby all "orbital" modes are sent to infinity. 


\section{BRANE WORLD GRAVITY IN ADS BLACK HOLE}

Next we have introduced a Planck brane as a UV cut-off at $r=r_{c}$ by reflecting the metric $\left(r \rightarrow r_{c} / r^{2}\right)$ at a $Z_{2}$ orbifold and adding a positive tension brane world action [4]. The essential new feature is that in addition to massive tensor glueballs, there is now a new normalizable tensor eigenfunction with zero mass the graviton. After scaling out the warp factor $g_{\mu \nu} \simeq r^{2}\left(\eta_{\mu \nu}+h_{\mu \nu}\right)$ the transverse tensor amplitude $h_{\mu \nu}^{\perp}=\epsilon_{\mu \nu}(p) T(r) e^{i p x}$, obeys the same equation as a minimally coupled scalar.

For comparison with the Randall-Sundram solution, we convert to the proper distance $y(r)$ from the horizon, $y(r)=\int_{b}^{r} d r / \sqrt{f(r)}$, and introduce an integrating factor, $\Psi=e^{-W(y)} T$, so that the tensor equation has the SUSY form,

$\left[\frac{\partial}{\partial y}-W^{\prime}(y)\right]\left[\frac{\partial}{\partial y}+W^{\prime}(y)\right] \Psi(y)=\frac{p^{2}}{r^{2}} \Psi(r)$

with prepotential $W(y)=-\frac{1}{2} \log [\sinh ((d+$ 1) $\left.\left.k\left(\left|y_{c}-y\right|-y_{c}\right)\right)\right]$. The effective potential $V_{\text {eff }}(y)=\left(W^{\prime}\right)^{2}-W^{\prime \prime}$ is plotted for $y \in\left[0, y_{c}\right]$ in the figure below.

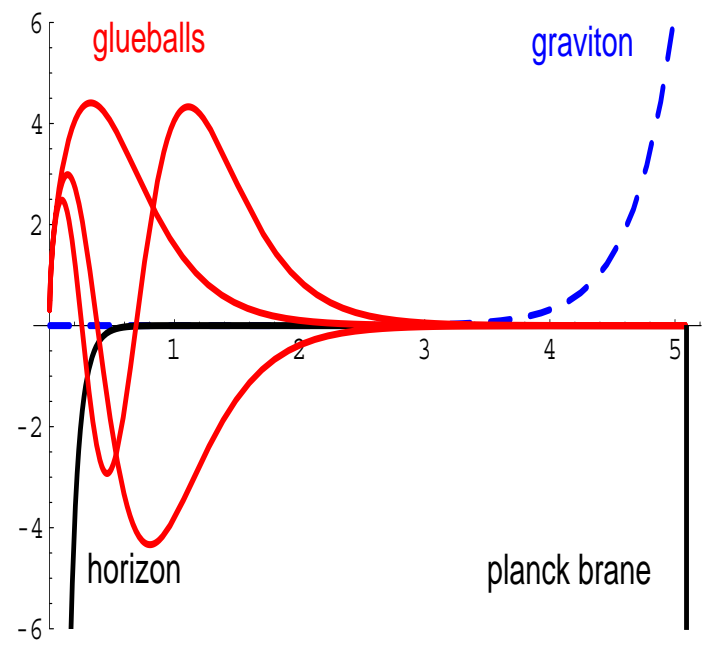

Note the "volcano" potential at the Planck brane is a delta function at the edge of the disk $\left(y_{c}=y\left(r_{c}\right)\right.$ for all $\left.\tau\right)$ with a single zero mass bound state (dashed line), the graviton wave function: $\Psi=\Psi_{0} \exp (-W(y))$. The black hole horizon at the origin $\left(y=0\right.$ or $\left.r=r_{\text {min }}\right)$ is rep- resented as a double pole in the potential $W(y)$ which localizes the massive glueball wave functions (solid lines). The mass hierarchy between the glueballs and the Planck mass is a reflection of the fact that glueball wave functions are concentrated near the black hole horizon where the graviton wave function is exponentially suppressed.

\section{REMARKS}

In addition to the graviton, careful analysis reveals a radion mode (flexing the proper distance separating the Planck brane from the black hole horizon) and a massless vector. At low energies the Planck brane action can be modified, consistent with covariant energy-momentum conservation, lifting these to small positive masses. If we were working in $A d S^{6}$, these three would be the only "domain wall" states added to the glueball spectrum. However the boundary of $A d S^{7}$ is $R^{3,1} \times S^{1} \times S^{1}$ with an extra flat compact $S^{1}$ along the 11th axis. Hence this analysis has in fact led us to 5 -d gravity with standard Kaluza Klein (KK) compactification and in addition to the graviton the usual KK gravi-photon and dilaton. At strong coupling there is an accidental $O(4)$ symmetry, causing for example the $2^{++} / 0^{++}$degeneracy seen in the spectrum (see Fig. 1). However this degeneracy will be broken at weak coupling since the 11-th axes is differentiate from the spatial axes as the $S^{1}$ on which the M-theory membrane is wrapped to get IIA strings. So the mechanism that lifts this symmetry must evidently give mass to the KK photon/dilaton as well.

\section{REFERENCES}

1. J. Maldacena, Adv. Theor. Math. Phys. (1998) 231.

2. E. Witten, Adv. Theor. Math. Phys (1998) 505.

3. R. C. Brower, S. D. Mathur, C-I Tan Nucl. Phys. B587 (2000) 249.

4. R. C. Brower, S. D. Mathur, C-I Tan, "Brane World Gravity and Glueballs in an AdS Black Hole" in preparation (2001). 